\title{
Design and Application of an Online English Self-learning Platform
}

\author{
https://doi.org/10.3991/ijet.v12.i08.7133 \\ Kun Liu \\ Qujing Normal University, Qijing, China \\ qjnuliukun@163.com
}

\begin{abstract}
This paper discusses the English learning methods and identifies the problems in English teaching and self-learning in China, and then based on the VB platform, it proposes building a platform to facilitate communication between teachers, learners and other participants. At the same time, it adopts the object-oriented development approach to analyze the construction method for the online English self-learning platform and uses UML language to design this platform. At last, this paper uses general testing methods to test the interfaces, functions, performances and security of the VB-based online English selflearning platform and analyzes the performances and deficiencies of the platform based on test results.
\end{abstract}

Keywords—online English; self-learning; B/S; system development

\section{Introduction}

Interest plays an essential role in English self-learning. Under the guidance of interest, students will take initiatives to search and learn English knowledge. Stimulated by strong interest, a student's nervous system will be very active, and their attention, active thinking and memory will be significantly improved, and as a result, they can receive information well and learn things better [1-3]. In order to achieve this purpose, this paper designs an online English self-learning platform, and discusses its application in detail.

English learning requires students to take the initiative to learn and have a strong interest in this language. The desire for knowledge can significantly improve students' learning efficiency. If students are aware of this and intentionally carry out such self-learning activities, they will achieve better results [4-6]. Under the selflearning model, students will have a more positive understanding of learning and develop a strong passion for knowledge, and in the process, their learning abilities and skills will also improve [7]. Whenever encountering any problem, students will tend to think by themselves rather than learn passively under the teacher's supervision under the traditional model. According to practical experience, this model can significantly increase students' interest and prevent them from getting tired of learning under the traditional learning model. Therefore, it is necessary to study the self-learning model. At present, there are many associated researches, but most of them focus on 
how students should learn English by themselves, master the appropriate learning skills and efficiently use some modern technology and equipment to help them doing self-study [8-11].

This paper mainly discusses the current research on online English self-learning, and then designs an online English learning platform based on VB platform so that students can use this online system to carry out self-learning and cultivate their independent learning abilities and further lay a good foundation for their language skills.

\section{Requirements for an Online English Self-learning Platform}

From the above analysis, we can see that an online English self-learning platform should have multiple functions. When building this kind of platform, we need to consider the following requirements [12-17]:

1. This platform should be built on the most current and advanced educational theories and avoid the shortcomings of the traditional education model. It is mainly built to cultivate students' self-learning abilities and provide convenience for students' exploratory learning.

2. On this platform, students can conveniently carry out repetitive learning and examine their learning results, and at the same time test their individual abilities in vocabulary, grammar, reading and listening. In addition, students can study the same contents as needed.

3. This platform should provide basic functions in self-learning. Students can develop their own learning schedule and contents on this platform with few restrictions. Teachers are mainly to check the learning progress of students and provide them with appropriate guidance and help

4. This platform should meet the needs of the learning network, so it has to be strong in both software and hardware and compatible on multiple operating systems. Besides, it should be easy to transplant and extend and able to process data efficiently.

\section{$3 \quad$ Analysis and Design of the Online English Self-learning Platform}

\subsection{Analysis of the System Operation Process}

After analyzing the requirement functions of this system, next we are going to analyze its operation process, and then design the system architecture and specific function modules. At the same time, we should fully analyze how to link the functions of the system together and determine the operation process to ensure the logicality of the system process. Different modules should be mutually independent and easy to link together, and the operation process of each module should be easy for users to understand. When developing this system, this paper considers the applicability and effectiveness of this system, designs the specific operation process and at the same time incorporates the software engineering idea to meet the needs of users. 
After analyzing the specific operations, we develop an operation process flow for this online platform, as shown in Fig. 1:

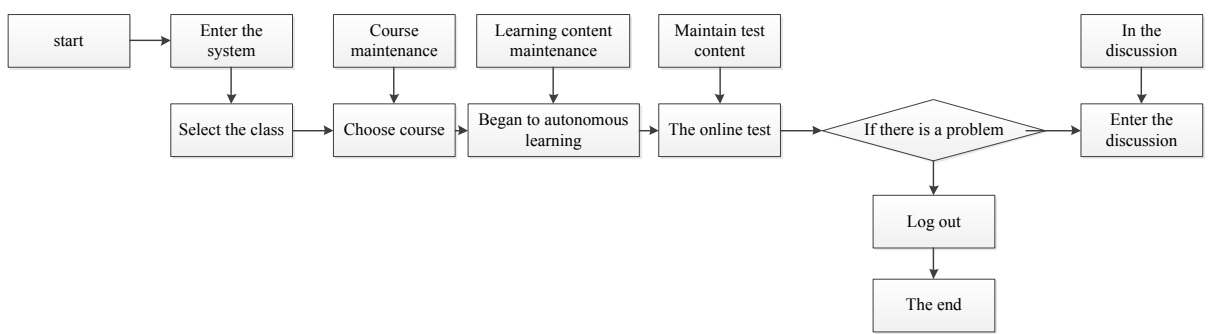

Fig. 1. Process Flow Chart of the Online English Self-learning Platform

\subsection{UML Modeling}

This modeling language is often used in the structured development. It is widely used in the system development field. This modeling language mainly uses some standard symbols to describe system roles and their relationships. Therefore, it is used to provide appropriate modeling and visual support for system development. During the analysis of system requirements, it is mainly used to describe the functional requirements of each role in the system. During the development, this paper uses UML to establish a use case model of the system, as shown in Fig. 2:

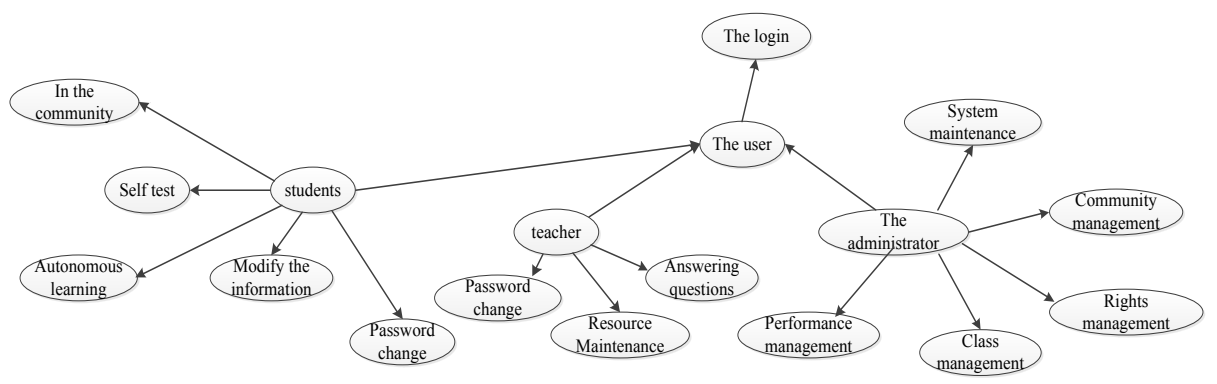

Fig. 2. Use Case Model of the Online English Learning Platform

This case use diagram clearly shows the roles of different users in this platform, but it does not describe the specific functions. Therefore, we need to develop a user model diagram for students according to the requirements of different users and describe the implementation process of the use case based on this model, as shown in Fig. 3.

From this diagram, we can clearly see the correspondences between all function modules and users and the functions that different users can realize through these modules, and based on this information, we determine the correlations between the business classes and the objects. 


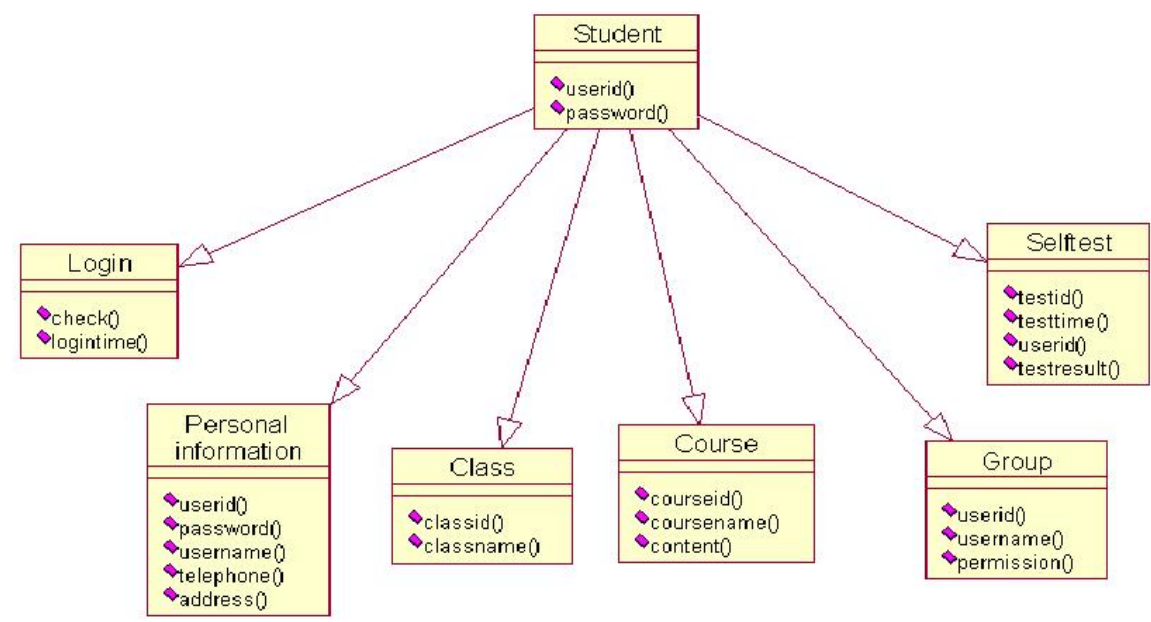

Fig. 3. Student Domain Model of the Online English Self-learning Platform

\subsection{System architecture design}

In order to make the system easier to use and expand its application scope, in the architecture design, the system chooses the $\mathrm{B} / \mathrm{S}$ architecture and builds the system architecture based on the VB platform. The browser used in this system can efficiently parse different types of codes without any barrier in application.

Under this architectural pattern, the system can be divided into three layers, including the presentation layer, the business logic layer and the data layer. The presentation layer is mainly used to display the processing results, and the data layer is mainly related to database operations. The system developed based on this architecture can easily realize a modular framework, and the modules are highly cohesive - different modules can easily transmit data through interfaces. Under this architecture pattern, all the user information is stored in the data layer, which ensures that the data is highly independent and secured. Users' operations are mainly carried out through the business logic layer, so that users will not come into contact with the data, and the results obtained will be sent to users through the presentation layer. In this case, users do not need to bear special resource burden; in other words, they can carry out operations with the minimum hardware of the presentation layer. All operations will go through the business logic layer. The advantage of this approach is to maximize the use of the resources on this layer, and the system updates, maintenance and modification are also achieved on this layer, providing great convenience for the system management and maintenance. However, this also increases the burden on the business logic layer, requiring good hardware and software performances. This architecture can also protect the security of users. Details of this architecture are shown in Fig. 4 and 5: 


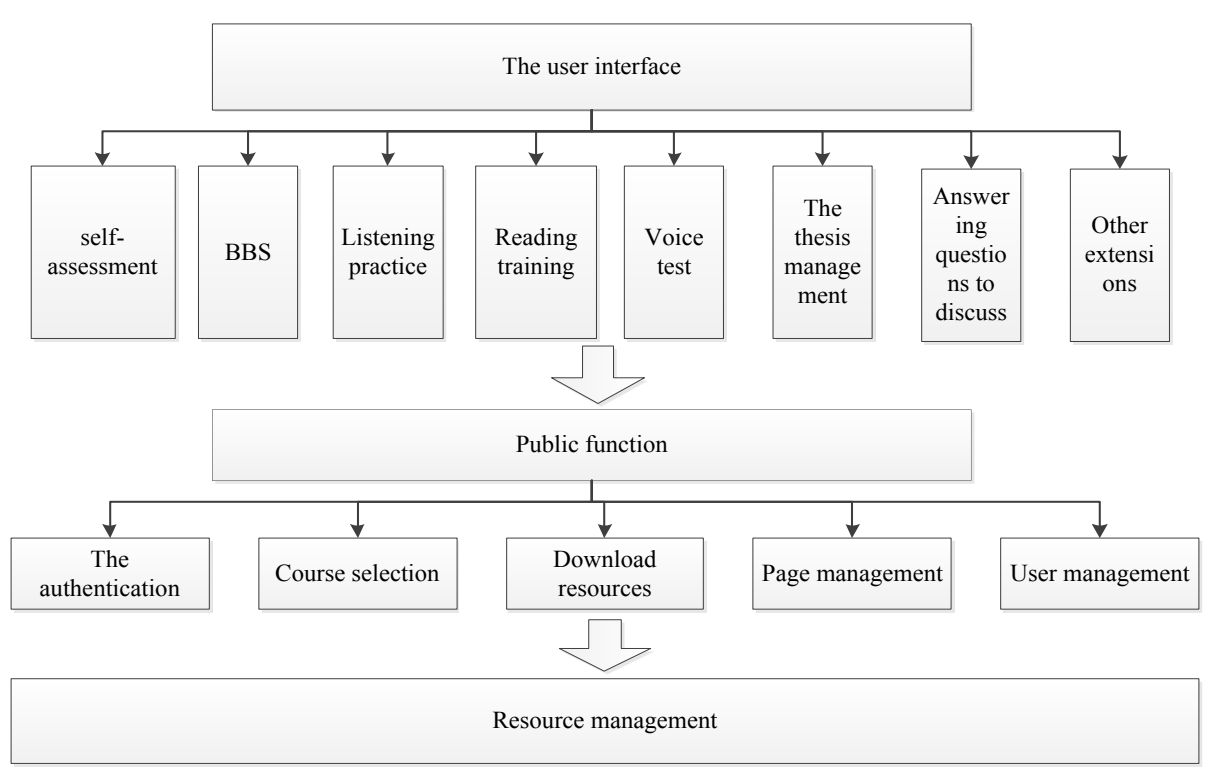

Fig. 4. Three-Layer B/S Architecture of the Online English Learning Platform

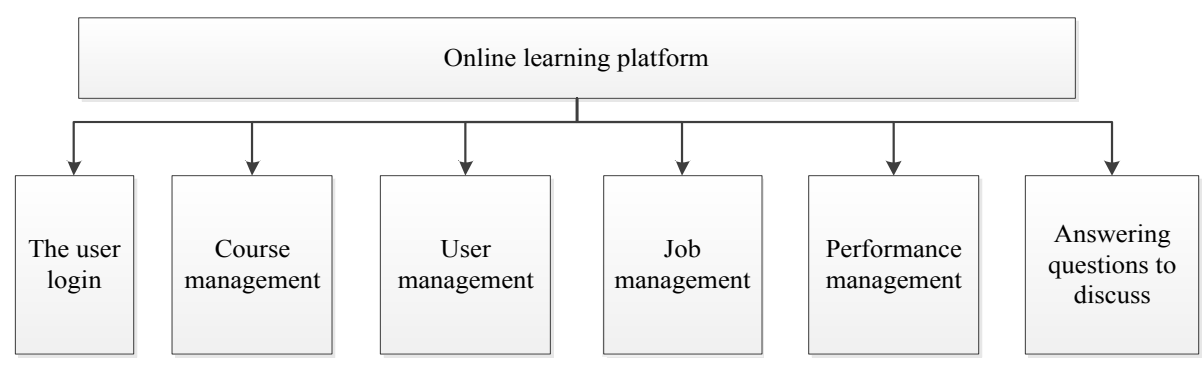

Fig. 5. Major Functions of the Online English Learning Platform

\subsection{System function module design}

The above is the detailed analysis of the functional requirements for the system. From the analysis results, we can see that users of this system include students, teachers and administrators, and their functional operations are mainly realized through the foreground and background of the system. These functional operations mainly include student information management, registration information management, class selection and course selection, etc. General users should be given these functions. They can easily find these function modules on the front page of this system. Course management, user management and rights management are mainly set up for users with higher authorities. For example, the system administrator usually uses the background management functions, and the teacher has more functions than students. We can also simply regard students and the administrator as the background and foreground users of the system. Details of the system outline design are shown in Fig. 6: 


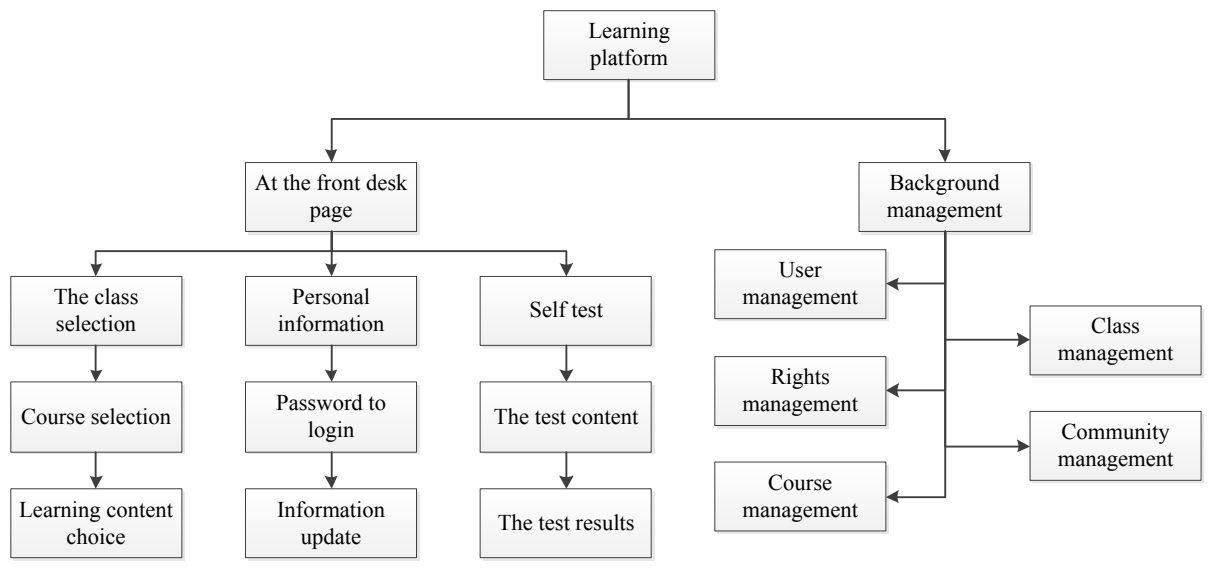

Fig. 6. Overall Functional Structure of the Online English Learning Platform

\subsection{System database design}

The operands of the system are various types of data. Under all circumstances, the system realizes the functions through data manipulation and processing, which requires the development of a corresponding database system. The user information, class information, course information, scores and homework are all stored in a certain data format. In order to better manage and manipulate these data resources, we need to design a system database to achieve these functions. At the same time, in order to efficiently integrate the modules in this system, the database system should be highly performing.

Considering the functional requirements for the system and the difficulties in development, when developing the database, we mainly use the business process flow chart to analyze the relationships between different data, then perform detailed analysis, and based on this, determine the data relations between different entities, mainly including one to many, one to one, and many to one relations. Then we design the database logic model for the system based on these relations, laying a good foundation for the establishment of the database system.

This paper performs detailed analysis of the database design for different function modules and the results are shown in Fig. 7. This can be regarded as a logic database model. At this point, the system design is completed. 


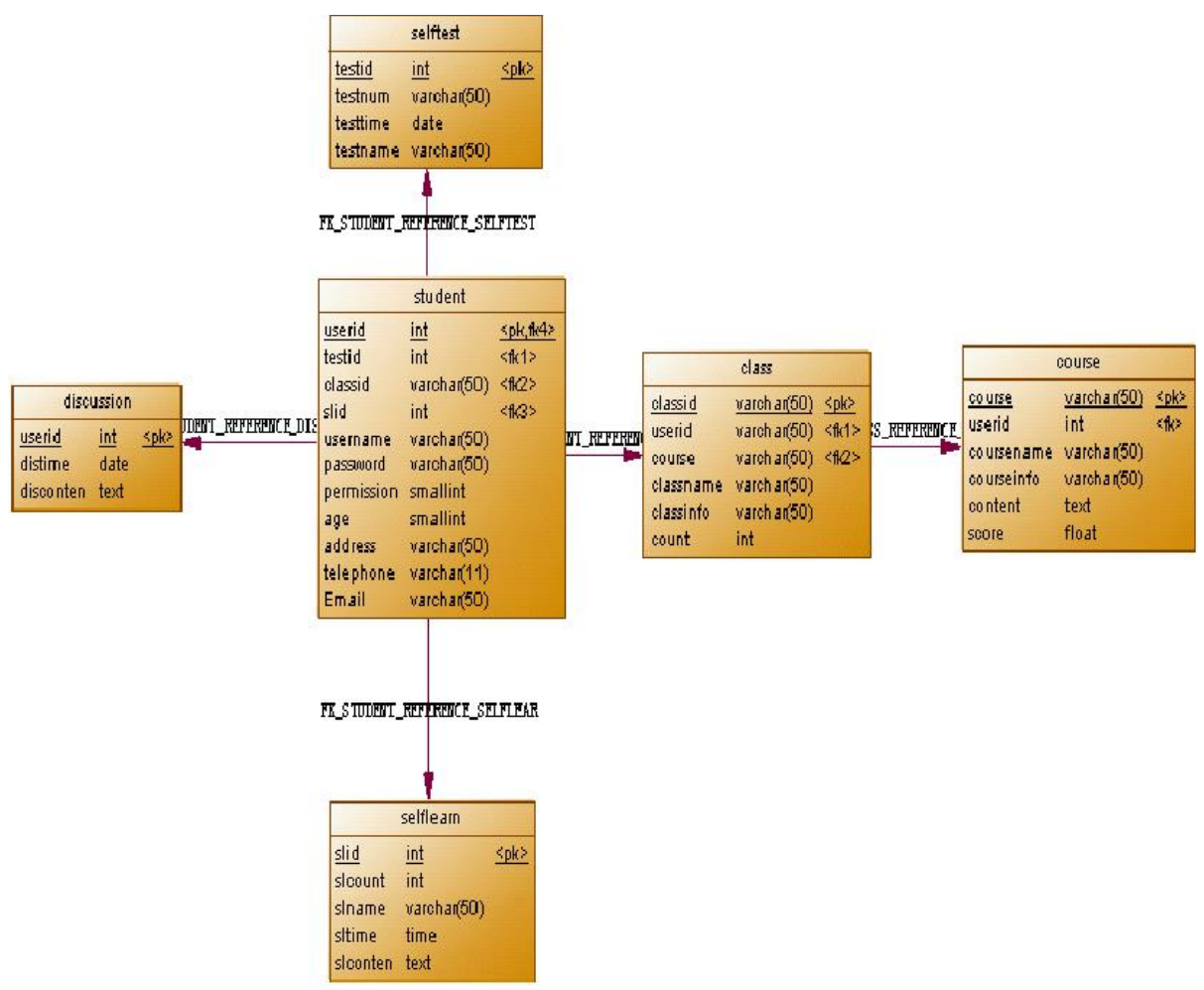

Fig. 7. Logical Database Model of the Online English Learning Platform

\section{System Test}

\subsection{Interface test}

System test is a major part of system development. By testing the functional performance of the system, we ensure that the purposes of the system are achieved. In the system test, we first test the system interface. The interface can realize the functions of the presentation layer. It is mainly used to directly interact with users and the user can obtain the data processing result through the interface. Users' direct impression about the software is also from the system interface. Therefore, we need to carefully design the system interfaces to make them easy to use and clear and concise. In addition, for users that are unfamiliar with the system, there should be functions like help and instructions on the interfaces to improve user satisfaction. The interface test mainly consists of individual test on each window to find out whether each of them is in line with the expected requirements and set up in accordance with standards. The user group of this learning platform is known, i.e. college students. They use this platform to learn English, so both Chinese and English will appear on the interfaces at the same time, which has raised certain requirements for the Chinese and English coding. If 
there is any coding error, gibberish will appear on the interface. In addition, considering the different typesetting of Chinese and English, if the same format is used, the interface will look messy. So in the interface test, we need to pay attention to literal encoding and format.

There are a number of requirements for the interfaces of this learning system. They should not only look nice but also be multi-functional. A lot of software realizes the functions through hyperlinks, so in the interface test, we also need to check whether the hyperlinks are correctly shown and whether the function modules are displayed in a rational manner.

\subsection{Functional test}

The functional test is mainly carried out to test the functions of the system, which generally does not take into account the internal code of the system; therefore, it can also be regarded as a black box test. This test is mainly carried out based on the software specifications and other documents. It is not technically demanding - the testers only need to input the test case according to the requirements and compare the test results. They do not need to understand how the system works. All they need to do is compare and analyze the output results and determine whether there is any functional defect in the software and whether the development objectives of the system are achieved.

We test all the function modules in this system according to the business process and determine whether the system functions are realized based on the output results of the test cases. And the detailed test results are listed in Tab. 1:

Table 1. Results of the Functional Test

\begin{tabular}{|l|l|l|}
\hline \multicolumn{1}{|c|}{ Test content } & \multicolumn{1}{c|}{ Expected results } & \multicolumn{1}{c|}{ Actual results } \\
\hline Normal login & Correct login & Equivalent to desired effect \\
\hline Login error & Unable to log in & Equivalent to desired effect \\
\hline Right into the class & Normal to enter & Equivalent to desired effect \\
\hline Error into the class & Unable to enter & Equivalent to desired effect \\
\hline Homework to download & Normal to enter & Equivalent to desired effect \\
\hline Homework uploading & Normal to enter & Equivalent to desired effect \\
\hline
\end{tabular}

\section{Conclusions}

This paper mainly analyzes the current status of online English learning and designs an online self-learning application platform based thereon. In the process of the research, this paper analyzes the requirements raised by the English educational reform in China, describes the current domestic and international researches on online self-learning, and then discusses in detail the importance of the self-learning method in applied learning. After that, it designs a learning platform based on the English self-learning practices. During design and development, it chooses the b/s architecture 
and the VB platform, utilizes the object-oriented software engineering development technology, analyzes the user requirements, feasibility and business process of this system, and builds a requirement model based on this, and then it designs and implements this learning platform. During the development, it considers the applicability and effectiveness of this platform to make sure it can satisfy users' needs. In order to meet the software quality requirement, this paper also incorporates the software engineering idea. At last, it carries out the interface and functional test on the system and analyzes the test results. The results show that the system has successfully achieved its development objectives and can satisfy users' needs.

\section{References}

[1] Jr, E.P.E.S., Idiart, M.A.P. (2004). Autonomous learning architecture for environmental mapping. Journal of Intelligent \& Robotic Systems, 39(3), 243-263. https://doi.org/10.1023/B:JINT.0000021023.56384.55

[2] Schiebener, D., Morimoto, J., Asfour, T., Ude, A. (2013). Integrating visual perception and manipulation for autonomous learning of object representations. Adaptive Behavior, 21(5), 328-345. https://doi.org/10.1177/1059712313484502

[3] Shen, W. M. (1993). Discovery as autonomous learning from the environment. Machine Learning, 12(1), 143-165. https://doi.org/10.1007/BF00993064

[4] Mugan, J., Kuipers, B. (2012). Autonomous learning of high-level states and actions in continuous environments. IEEE Transactions on Autonomous Mental Development, 4(1), 70-86. https://doi.org/10.1109/TAMD.2011.2160943

[5] Leclercq, E., Druaux, F., Lefebvre, D., Zerkaoui, S. (2005). Autonomous learning algorithm for fully connected recurrent networks. Neurocomputing, 63, 25-44. https://doi.org/10.1016/j.neucom.2004.04.007

[6] Snodin, N.S. (2013). The effects of blended learning with a cms on the development of autonomous learning: a case study of different degrees of autonomy achieved by individual learners. Computers \& Education, 61(1), 209-216. https://doi.org/10.1016/j.compedu.2012. $\underline{10.004}$

[7] Moriyama, T., Gunji, Y.P. (1997). Autonomous learning in maze solution by octopus. Ethology, 103(103), 499-513. https://doi.org/10.1111/j.1439-0310.1997.tb00163.x

[8] Piastra, M. (2013). 2013 special issue: self-organizing adaptive map: autonomous learning of curves and surfaces from point samples. Neural Networks, 41(6), 96-112. https://doi.org/10.1016/j.neunet.2012.07.007

[9] Di, N.A., Marocco, D., Di, N.S., Cangelosi, A. (2013). Autonomous learning in humanoid robotics through mental imagery. Neural Networks, 41(5), 147.

[10] Geiger, C.D., Uzsoy, R., Aytuğ, H. (2006). Rapid modeling and discovery of priority dispatching rules: an autonomous learning approach. Journal of Scheduling, 9(1), 7-34. https://doi.org/10.1007/s10951-006-5591-8

[11] Bianchi, R.A., Ribeiro, C.H., Costa, A.H. (2008). Accelerating autonomous learning by using heuristic selection of actions. Journal of Heuristics, 14(2), 135-168. https://doi.org/10.1007/s10732-007-9031-5

[12] Bagnell, J.A., Bradley, D., Silver, D., Sofman, B. (2010). Learning for autonomous navigation. IEEE Robotics \& Automation Magazine, 17(2), 74-84. https://doi.org/10.1109/MRA.2010.936946 
[13] Fox, M. (2012). Plan-based policy-learning for autonomous feature tracking. Autonomous Robots, 37(1).

[14] Billard, A., Hayes, G.M. (1999). Drama, a connectionist architecture for control and learning in autonomous robots. Adaptive Behavior, 7(1), 35-63. https://doi.org/10.1177/ 105971239900700103

[15] Siomau, M. (2013). A quantum model for autonomous learning automata. Quantum Information Processing, 16(5), 1211-1221.

[16] Gómez, M. (2012). Optimal motion planning by reinforcement learning in autonomous mobile vehicles. Robotica, 30(2), 159-170. https://doi.org/10.1017/S0263574711000452

[17] Huang, L.H. (2016). Gm(0,n)model -based analysis of the influence factors of network english learning platform. Journal of Grey System, 19.

[18] Bourbakis, N.G. (1989). Heuristic collision-free path planning for an autonomous platform. Journal of Intelligent \& Robotic Systems, 1(4), 375-387. https://doi.org/10.1007/BF00126467

\section{$7 \quad$ Author}

Kun Liu, male, Qujing, Yunnan, China, Han ethnicity, master and lecturer of computer education, artificial intelligence, etc. Starting to work in 2002, he is mostly engaged in educating undergraduates in the field of computer science, teaching the courses of Computer organization, The C Programming Language, Object-Oriented Programming, Fundamentals of Computer Science and Technology, Computer Fundamentals, etc. Over the years, he has presided over 1 university-level project, participated in 3 provincial projects, published 20 papers and co-edited a textbook. He is with the School of Information Engineering, Qujing Normal University, Qijing 655011, China (qjnuliukun@163.com)

Article submitted 10 May 2017. Published as resubmitted by the author 15 June 2017. 